\title{
Transactive Energy for Flexible Prosumers Using Algorithmic Game Theory
}

This paper was downloaded from TechRxiv (https://www.techrxiv.org).

\section{LICENSE}

CC BY-NC-SA 4.0

SUBMISSION DATE / POSTED DATE

06-02-2021 / 08-02-2021

\section{CITATION}

Tsaousoglou, Georgios; Pinson, Pierre; Paterakis, Nikolaos (2021): Transactive Energy for Flexible Prosumers Using Algorithmic Game Theory. TechRxiv. Preprint.

https://www.techrxiv.org/articles/preprint/Transactive_Energy_for_Flexible_Prosumers_Using_Algorithmic_Gam 


\title{
Transactive Energy for Flexible Prosumers Using Algorithmic Game Theory
}

\author{
Georgios Tsaousoglou, Pierre Pinson, Fellow, IEEE, and Nikolaos G. Paterakis, Member, IEEE
}

This paper is accepted for publication in IEEE Transactions on Sustainable Energy. (C) 2021 IEEE. Personal use of this material is permitted. Permission from IEEE must be obtained for all other uses, in any current or future media, including reprinting/republishing this material for advertising or promotional purposes, creating new collective works, for resale or redistribution to servers or lists, or reuse of any copyrighted component of this work in other works.

\begin{abstract}
In modern smart grids, the focus is increasingly shifted towards distributed energy resources and flexible electricity assets owned by prosumers. A system with high penetration of flexible prosumers, has a very large number of variables and constraints, while a lot of the information is local and nonobservable. Decomposition methods and local problem solving is considered a promising approach for such settings, particularly when the implementation of a decomposition method features a market-based analogy, i.e. it can be implemented in a Transactive Energy fashion. In this paper we present an auction-theoretic scheme for a setting with non-convex prosumer models and resource constraints. The scheme is evaluated on a particular case study and its scalability and efficiency properties are tested and compared to an optimal benchmark solution. A game-theoretic analysis is made with respect to how an intelligent agent, that bids on behalf of a prosumer can try to strategize within the auction, in order to make itself better-off. Our simulations show that there is an alignment of incentives, i.e., when the prosumers try to strategize, they actually improve the auction's efficiency.
\end{abstract}

Index Terms-flexibility, Transactive Energy, Algorithmic Game Theory, Auction

\section{INTRODUCTION}

$I^{\prime}$ $\mathrm{N}$ modern power systems there is a profound trend towards investing in electricity assets located at the edge of the network [1]. These include batteries, smart appliances, electric vehicles, micro-generation etc. Such flexibility assets, owned by prosumers, create several challenges regarding their integration in the electricity markets and the network management algorithms. In contrast to power plants, the local constraints of each prosumer (and its flexibility assets) are not visible to the operator and they can also be changing from one day to another. Thus, when it comes to energy management, there is a general consensus that local problem solving is necessary [2], [3]. Naturally, in a distributed optimization where each prosumer solves a local problem, certain coordination is needed in order to satisfy global constraints (i.e. constraints that involve more than one prosumer). Such constraints can relate to the physical flows of the electrical grid or to capacity limits of system resources. For example, the operator of the distribution

Georgios Tsaousoglou received funding from the European Union's Horizon 2020 research and innovation programme under the Marie Skłodowska-Curie grant agreement No.754462

G. Tsaousoglou, and N.G. Paterakis are with the Eindhoven University of Technology. P. Pinson is with the Technical University of Denmark. network can ex-ante allocate an upper limit of network capacity that is available to a community of prosumers, for reasons of managing congestion in the distribution grid, as explained in [4]. Also, the capacity of a distribution network transformer is another type of a constraint that jointly involves multiple prosumers [5]. These types of constraints are generally referred to as resource constraints.

\section{A. Motivation}

Managing and coordinating a set of hundreds, or thousands of prosumers creates an important scalability barrier and the research community has been trying to build efficient and scalable algorithms for the energy management and global constraint satisfaction problem. Several decomposition methods have been proposed in the recent literature, where prosumer decisions are made locally and the coordination is typically achieved by the iterative exchange of energy prices. This framework is often referred to as Transactive Energy [6]. However, most such methods can only accommodate convex problems; hence, a convexity assumption is very common in the relevant literature. While convex models facilitate model analysis and strong theoretical results, the extent to which they can realistically model the prosumers' flexibility assets is often deemed quite limited.

On the other hand, the non-convex nature of asset models makes it extremely difficult to obtain decomposition methods with optimality guarantees for allocating energy among a community's prosumers. Even achieving global feasibility alone, is reported as a quite challenging issue [7]. A few theoretical tools are available (e.g. Bender's decomposition) but those are not suitable to be implemented in a marketoriented framework, i.e., with Bender's decomposition the local information is communicated to a central node and the centralized optimization problem is decomposed to multiple cores for computational reasons. This method is not compatible with a Transactive Energy framework where prices are communicated to the prosumers and the latter make their energy management decisions locally.

Another important issue arises with respect to incentivizing the prosumers to make their local decisions in accordance to some social objective (e.g. minimizing the operational 
cost of the system or accommodating global constraints). Market frameworks can be very efficient in this aspect, but, if not designed carefully, they can also be prone to strategic participant behavior and a consequent vast inefficiency or even a plain failure.

Such aspects become increasingly relevant, since developments in computer science, and particularly in Artificial Intelligence (AI), offer the necessary tools for designing intelligent agents that can make decisions on behalf of the prosumer. Such intelligent agents have already been proposed in the smart grid literature, for home energy management systems [8], and for controlling the behavior of smart residential appliances [9]. An intelligent agent is programmed to optimized a payoff function. A recurring phenomenon in state of the art AI programs is that the agent discovers unexpected ways to optimize the given payoff, which the human designers did not foresee. An example of this phenomenon is the infamous bot in [10], that was told to minimize the contact time between its feet and the ground. The goal was to make the bot walk faster, but it ended up learning to walk on its elbows. Another recent example refers to the creative ways discovered by the bots of OpernAI, to leverage the model's "physics" and optimize their play in a hide-and-seek game [11]. Thus, with intelligent agents making decisions on behalf of prosumers, strategic behavior can also occur even without such intention from the prosumer's side.

Because of these phenomena, it is important to be very careful in the design of an energy management system with distributed decision-making by intelligent agents. Especially in the community context, where the reach of market monitoring and auditing procedures is naturally limited, intelligent agents with computational capabilities, acting on behalf of prosumers, can find an inviting place to exploit. Based on the previous observations, one can identify some important requirements for market-based community energy management algorithms:

- Scalability: supporting local decision-making and integrating large numbers of prosumers and their flexibility assets in a reasonable computational time.

- Efficiency: coordinating prosumer decisions by providing suitable market signals, so as to achieve a social objective and satisfy global constraints.

- Incentives: designing a market framework that is strategyproof and cannot be gamed/manipulated by intelligent agents that participate on behalf of prosumers.

- Non-convexities: taking into account the non-convex nature of realistic flexibility assets' models.

\section{B. Related Work}

Along with high penetration of flexibility assets in the electricity system, the issue of scalability has been the focus of several studies. Reference [12], presents a literature review on distributed optimization techniques applied to modern smart grids. In [5], a resource constraint is considered by taking into account the limit of a community's aggregated energy exchange through the distribution transformer. In [13] a P2P trading scheme is proposed where the transactions have to be approved by the operator, in order to ensure the satisfaction of global constraints. In [14], the alternate direction method of multipliers (ADMM) is used to solve the energy management problem of a community of prosumers. Global resource constraints were considered in the form of limiting the community's import energy due to contracted peak-shaving services. However, the local cost functions of the prosumers are assumed to be convex. In [15] the ADMM approach is applied to a system with non-convex models, although in this case the satisfaction of global constraints is not guaranteed.

Configuring decomposition methods with market frameworks (i.e. typically by interpreting Lagrange multipliers as price signals), is a well-established analogy that has also been applied in various use cases. A typical example of such studies is [16], while in [17] global network constraints are also considered. However, these studies also assume convex local cost functions of prosumers and their flexibility assets. A similar approach is proposed in [18], in a use case with non-convex user preferences but, again, there is no guarantee of global constraint satisfaction. Techniques from Artificial Intelligence have been proposed in order to handle nonconvex energy management problems (e.g. actor-critic deep reinforcement learning [19] and temporal difference learning [20]). However, it is an open and challenging problem for such methods to guarantee global constraint satisfaction.

At the same time, the potential of Artificial Intelligence techniques towards making local energy management decisions on behalf of a prosumer [9], or also bidding on behalf of a community, in an electricity market [21] has been demonstrated. In [22], however, it is shown (through bilevel optimization) that strategic bidding by intelligent agents, acting on behalf of prosumers, can compromise the system's efficiency. In order to account for strategic behavior, gametheoretic aspects need to be considered [23]. In [23] a VickreyClarke-Groves (VCG) mechanism is proposed in order to incentivize truthful participation in the market, while [24] proposes the same mechanism for offering Demand Response services. The mechanism is applied to a low-voltage network (accounting also for power flow constraints) in [25]. In [26], distributed mechanism design is used to achieve the same incentive alignment in a peak-shaving use case, while also exhibiting attractive scalability properties, in contrast to the standard VCG mechanism. A decomposition method for accommodating coupling constraints in a game-theoretic framework is shown in [27], while in [28] a novel mechanism is proposed for satisfying resource constraints in a setting with strategic, price-anticipating prosumers. Finally, study [29] proposes a generalization of the payment rule used by VCG, in order to maintain truthful participation also in the case that the DSO opts for a max-min fair allocation for the distribution network resources, and not only for an allocation that maximizes the social welfare. Nevertheless, all the studies of this paragraph again assume convexity of prosumer models.

Overall, each of the above studies addresses a subset of the the four requirements discussed in the introduction. A summary of the literature review is presented in Table I.

\section{Contributions and Organization}

In this paper, we draw on concepts of algorithmic game theory and propose an auction-theoretic solution for a non- 
TABLE I

CLASSIFICATION OF LITERATURE BASED ON REQUIREMENTS

\begin{tabular}{|c||c|c|c|c|}
\hline & $\begin{array}{c}\text { Scalab- } \\
\text { ility }\end{array}$ & $\begin{array}{c}\text { Efficiency \& global } \\
\text { constraint satisfaction }\end{array}$ & $\begin{array}{c}\text { Incentives \& } \\
\text { strategic behavior }\end{array}$ & $\begin{array}{c}\text { Non-convex } \\
\text { models }\end{array}$ \\
\hline \hline$[5],[13]$ & $\boldsymbol{V}$ & $\boldsymbol{V}$ & $\times$ & $\boldsymbol{\checkmark}$ \\
\hline$[14],[15]$, & $\boldsymbol{V}$ & $\boldsymbol{V}$ & $\times$ & $\times$ \\
{$[16],[17]$} & & & $\times$ & $\boldsymbol{\checkmark}$ \\
\hline$[18]-[20]$ & $\boldsymbol{V}$ & $\times$ & $\boldsymbol{V}$ & $\times$ \\
\hline$[23]-[25]$ & $\times$ & $\boldsymbol{V}$ & $\boldsymbol{V}$ & $\times$ \\
\hline$[26]-[28]$ & $\boldsymbol{V}$ & $\boldsymbol{V}$ & $\boldsymbol{V}$ & $\boldsymbol{V}$ \\
\hline This work & $\boldsymbol{V}$ & $\boldsymbol{V}$ & & \\
\hline
\end{tabular}

convex community energy management problem with (global) resource constraints. We test the proposed method on a particular case study and conduct simulations to assess its performance with respect to all four of the above requirements. To the best of the authors' knowledge, this is the first study to consider all four of the requirements of community energy management systems. In particular, the proposed method can handle non-convex prosumer models and maintain its global feasibility guarantees, while leaving no room for strategic participant behavior. Our simulation results assess the method's efficiency and scalability properties.

The remainder of this paper is organized as follows: Section II presents the system model and the problem formulation as well as a couple of counter-examples of how commonly proposed approaches can fail to satisfy certain requirements. Section III presents the proposed approach. In Section IV, a case study is modeled with prosumers featuring thermostatically controlled loads, electric vehicles, storage and local micro-generation. In Section V, the proposed solution is applied to the case study and the results are presented. Finally, Section VI concludes the paper.

\section{System Model And Problem Formulation}

We model a community of prosumers, where each prosumer features a set of controllable flexibility assets. The set of prosumers is denoted by $N$, where each prosumer bids for electricity for a time horizon $T$. The prosumer's local information includes the models of the prosumer's flexibility assets, local constraints and user preferences.

For a prosumer $n \in N$, let $A_{n}$ denote the set of $n$ 's flexibility assets and $y_{m}^{t}(\mathrm{kWh})$ denote the controllable power profile of asset $m$ in timeslot $t$. The prosumer's aggregated profile in $t$ (from all its flexibility assets) is denoted by $x_{n}^{t}$ $(\mathrm{kWh})$. It is

$$
\sum_{m \in A_{n}} y_{m}^{t}=x_{n}^{t}
$$

Since in this paper we mainly refer to prosumers of residential/commercial buildings, we adopt the convention of generally referring to $x_{n}^{t}$ as the prosumer's consumption. Let $\boldsymbol{x}_{n}=\left\{x_{n}^{t}\right\}_{t \in T}$ denote a consumption profile of prosumer $n$ throughout the horizon $T$. The locally feasible set of $\boldsymbol{x}_{n}$ is denoted as $L_{n}$ and is defined by a set of local constraints on $\boldsymbol{x}_{n}$. The set of local constraints $L_{n}$ is defined by the operational characteristics and models of the prosumer's flexibility assets (e.g. asset operational points, efficiency of power exchange of a battery etc). Set $L_{n}$ is non-convex since constraints typically

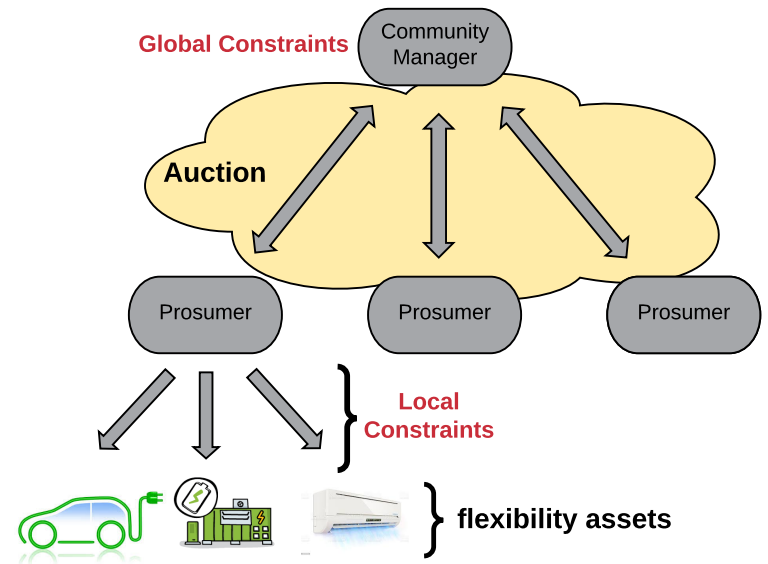

Fig. 1. System's architecture

include binary variables, e.g., for controlling the operation of flexibility assets that operate in discrete power levels. Also, intertemporal constraints may apply, where an asset's power $y_{m}^{t}$ at timeslot $t$ is dependent to the power $y_{m}^{t-1}$ of the asset on the previous timeslot $t-1$.

User preferences and asset operational costs are modeled via a cost (or disutility) function $c_{n}\left(\boldsymbol{x}_{n}\right): \mathbb{R}^{|T|} \rightarrow \mathbb{R}$ that maps a power profile $\boldsymbol{x}_{n}$ to a certain cost value for $n$.

On top of local constraints $\boldsymbol{x}_{n} \in L_{n}$, there is a set $G$ of community coupling constraints. These global constraints relate the power profile of more than one prosumer, such as limits on the community's aggregated consumption. A graphic representation of the system's architecture is presented in Fig. 1. Formally, it is required that the community's aggregated profile $\boldsymbol{X}_{N}=\left\{X_{N}^{1}, X_{N}^{2}, \ldots, X_{N}^{|T|}\right\}$, where $X_{N}^{t}=\sum_{n \in N} x_{n}^{t}$, satisfies the set of global constraints, i.e., $\boldsymbol{X}_{N} \in G$. Set $G$ can be non-convex for our purposes, however we make the following Assumption

Assumption 1. There is a set of threshold values $\left\{\overline{\mathrm{x}}_{n}^{t}\right\}_{n \in N, t \in T}$, for which it is

$$
\left\{\boldsymbol{X}_{N}: x_{n}^{t} \leq \overline{\mathrm{x}}_{n}^{t}, \forall n \in N, t \in T\right\} \in G
$$

Intuitively, Assumption 1 expresses the mild condition that if all prosumers reduce their consumption enough, the remaining (inflexible) demand is feasible for the system to satisfy.

Finally, the electricity cost of the community's energy in timeslot $t$ is denoted as $g^{t}\left(X_{N}^{t}\right)$. The objective of the community is to find a set of vectors $\boldsymbol{x}_{n}$, that are locally and globally feasible, and also minimize the community's system cost:

$$
\begin{array}{cl}
\min _{\boldsymbol{x}_{n}}\left\{\sum_{n \in N} c_{n}\left(\boldsymbol{x}_{n}\right)+\sum_{t \in T} g^{t}\left(X_{N}^{t}\right)\right\} \\
\text { s.t. } \quad & \boldsymbol{x}_{n} \in L_{n} \quad \forall n \in N \\
& \boldsymbol{X}_{N} \in G \\
& X_{N}^{t}=\sum_{n \in N} x_{n}^{t}
\end{array}
$$


Problem (2) is a non-convex problem and, depending on the form of functions $c_{n}\left(\boldsymbol{x}_{n}\right)$, it can be arbitrarily hard to solve it efficiently or even find a feasible solution. Moreover, cost functions $c_{n}\left(\boldsymbol{x}_{n}\right)$ and locally feasible sets $L_{n}$ are private to prosumer $n$ and the latter may not be eager to share this information with a central entity (or with other prosumers).

\section{A. Maintaining a per-timeslot price: a counter-example}

Since there is currently no general method for reaching an optimal solution to a distributed non-convex problem such as (2), the research community in power systems typically opts for an approach where a community energy manager iteratively sets an electricity price $\lambda^{t}$ for each timeslot (e.g. [4], [30]) and the prosumers respond by communicating their profiles $\boldsymbol{x}_{n}$ for the current set of prices. If the global constraints are not satisfied, the community manager increases the prices accordingly and the procedure iterates until the global constraints are satisfied. For models with continuous variables, this approach is represented by Lagrangian relaxation methods. However, when this procedure is used in a setting with intelligent agents acting on behalf of prosumers, there is a clear incentive for each prosumer to strategically misreport its "optimal" profile along the algorithm's iterations.

1) Numerical example and truthful case: For the sake of demonstration, consider a setting where we have 3 prosumers who are bidding for energy consumption in 4 timeslots. Prosumer 1 requires $2 \mathrm{kWh}$ in timeslot 1 , while prosumer 2 requires $2 \mathrm{kWh}$ in timeslot 2. Prosumer 3 needs a total of $4 \mathrm{kWh}$ that have to be split in two consecutive timeslots, with $2 \mathrm{kWh}$ per timeslot. Prosumer 3 prefers to consume energy in timeslots 1 and 2, but can also satisfy its demand in timeslots 3 and 4, albeit with some disutility. The available energy is only $2 \mathrm{kWh}$ in each timeslot.

Using the approach described above, the community manager would begin with zero price in all timeslots. In the first iteration, prosumer 3 would opt for timeslots 1 and 2 (because it prefers them). The demand would be higher than the supply, so the prices in timeslots 1 and 2 would increase. This would repeat until the prices in timeslots 1 and 2 are high enough for prosumer 3 to migrate its demand to timeslots 3 and 4 , assuming that prosumers 1 and 2 are less elastic (are willing to pay more) than prosumer 3 , and thus prosumer 3 is the one who migrates its demand first. The procedure would terminate with increased prices in timeslots 1 and 2.

2) Strategic case: Now suppose that prosumer 1 acts strategically and only declares demand in timeslot 4 , although it does not wish to consume energy in this timeslot. Prosumers 2, 3 still compete for energy in timeslot 2 and increase the price, whereas in timeslots 1,3 and 4 prices remain zero. When prosumer 3 inevitably migrates to timeslots 3,4 , prosumer 1 observes the price increase and abandons timeslot 4 to declare its true demand, i.e., the need for consuming energy in timeslot 1 . The procedure terminates with prosumer 1 receiving energy in timeslot 1 , but this time at zero price. Intuitively, a prosumer would be better-off suppressing its reported energy consumption in the timeslots it is interested in, so that prices don't rise in the early iterations. Evidently, this misalignment of incentives can cause problems when all prosumers have an incentive to misreport their true preferences.

\section{B. The computational cost of truthfulness}

In order to tackle the problem of misreporting, we can draw on concepts of mechanism design. In particular, the unique mechanism that guarantees truthful participation and achieves optimality, is the Vickrey-Clark-Groves (VCG) mechanism. By applying the VCG mechanism, the community manager requests each prosumer to report its local cost functions $c(\cdot)$ and constraints $L$. The community manager then solves problem (2) in order to find the optimal allocation. After that, it solves problem (2) another $|N|$ times to find the prosumer payments. Specifically, each time it removes one prosumer from the problem and calculates the system cost of other prosumers, with and without the said prosumer. By subtracting the latter from the former, the payment of the prosumer is calculated [25]. With these payments, it is theoretically proven that it is to the best interest of every prosumer to truthfully report its local parameters. However the VCG mechanism is computationally costly and suffers from scalability issues, since it needs to solve $|N|+1$ optimization problems.

In what follows we provide a decomposition method, inspired by auction theory, that guarantees a globally feasible solution. Moreover, the auction is especially designed in such a way, that the prosumers' incentives are aligned with the social objective (as shown by simulation in Section V), while the algorithm's computational time and scalability are dramatically improved in comparison to the VCG mechanism.

\section{Proposed Solution}

In this section we adapt and modify a combinatorial auction originally proposed in $[31]^{1}$, to the problem described in the previous section. We assume a community manager that iteratively gathers the updated profiles $\boldsymbol{x}_{n}$ of all prosumers and is aware of the global constraints $\boldsymbol{X}_{N} \in G$. It is noted that the profile aggregation and the satisfaction of the global constraints can also be performed in a distributed fashion, through the use of Data Hash Tables as done in [26], without the need of a community manager. However, the implementation of such a protocol is an orthogonal problem which is out of scope for this paper.

Each prosumer $n$ bears a maximum consumption level $\mathrm{x}_{n}^{\max }$, such that $x_{n}^{t} \leq \mathrm{x}_{n}^{\max }, \forall t \in T$. Prosumer $n$ may also have non-flexible loads, that constitute a minimum profile $\mathbf{x}_{n}^{\min }$. In the proposed auction, each prosumer will maintain a bid $b_{n} \in \mathbb{R}$ (in monetary units), that represents $n$ 's total payment. It is highlighted here, that $b_{n}$ is a payment that corresponds to $n$ 's whole profile $\boldsymbol{x}_{n}$.

\footnotetext{
${ }^{1}$ The model presented in [31] is proposed for a setting with discrete items where participants have valuations for different item allocations that they receive. More specifically, [31] considers participants that desire one specific and publicly known allocation, or participants that desire different allocations but have the same valuation among those. In contrast, in this paper we consider the more general problem of allocating a continuous resource (i.e. energy) to a number of participants that have different valuations for different (not publicly known) allocations.
} 
The process begins by setting the requested demand for each prosumer to $x_{n}^{t}=\mathrm{x}_{n}^{\max }$ and initializing each prosumer's bid $b_{n}$ to zero. Also, a set of currently "winning" prosumers (i.e., prosumers that have been granted an allocation), denoted as $W$, is initialized to the empty set. At iteration $k$, the community manager iterates through the prosumers in decreasing order of their bids. If the previous profile $\boldsymbol{x}_{n}^{(k-1)}$ of prosumer $n$, is compatible with the aggregated profile of prosumers already in set $W$, i.e., $\boldsymbol{X}_{W^{(k)}}+\boldsymbol{x}_{n}^{(k-1)} \in G$, then $n$ is added to set $W^{(k)}$. Prosumer $n$ 's profile is set equal to the previous value, $\boldsymbol{x}_{n}^{(k)}=\boldsymbol{x}_{n}^{(k-1)}$, and it is added to the aggregated profile $\boldsymbol{X}_{W^{(k)}}$ of set $W^{(k)}$. If $n$ 's choice is not feasible, then the community manager provides $n$ with the aggregated vector $\boldsymbol{X}_{W^{(k)}}$ of the rest of the prosumers in $W^{(k)}$, and with the global constraints $\boldsymbol{X}_{W^{(k)}}+\boldsymbol{x}_{n}^{(k)} \in G$. Prosumer $n$, is required to compute a different vector $\boldsymbol{x}_{n}^{(k)}$, that is compatible with the profiles of the prosumers that are already in $W$, by solving the following optimization problem:

$$
\begin{aligned}
\boldsymbol{x}_{n}^{(k)}=\operatorname{argmin}\{ & \left.c_{n}\left(\boldsymbol{x}_{n}^{(k)}\right)+s_{n}\left(\boldsymbol{x}_{n}^{(k)}, \sum_{t \in T} g\left(X_{W^{(k)}}^{t}\right)\right)\right\} \\
\text { s.t. } \quad & \boldsymbol{x}_{n}^{(k)} \in L_{n} \\
& \boldsymbol{X}_{W^{(k)}}+\boldsymbol{x}_{n}^{(k)} \in G \\
& x_{n}^{t,(k)} \leq x_{n}^{t,(k-1)}, \forall t \in T
\end{aligned}
$$

where function $s_{n}(\cdot)$ captures the social contract that determines $n$ 's share of the total community cost. Intuitively, the algorithm maintains the satisfaction of global constraints, by requiring the profile of each prosumer $n$ that is about to enter the set $W$, to be compatible with the profiles of prosumers that played prior to $n$ and are already in $W$. Also, the last constraint in (3) obligates the prosumer's profile in each timeslot to be monotonically decreasing across the auction iterations. This prevents the auction from oscillating among globally infeasible solutions, without making any progress. In case $n$ was not able to find a feasible solution within a predetermined time, then, at the end of the iteration, $n$ can increase its bid $b_{n}$ and maintain its previous profile, i.e. $\boldsymbol{x}_{n}^{(k)}=\boldsymbol{x}_{n}^{(k-1)}$. In the next iteration all prosumers in $W$ will be transferred to set $P$, and set $W$ will be reinitialized. Thus, $n$ will be prioritized (since it will have a higher bid) and it will be easier for $n$ to find a feasible solution, since $\boldsymbol{X}_{W}$ will be smaller (or empty) upon $n$ 's turn. If $n$ could not find a feasible solution and also cannot increase its bid, because it will get higher than the prosumer's current cost $c_{n}\left(\boldsymbol{x}_{n}^{(k)}\right)+s_{n}\left(\boldsymbol{x}_{n}^{(k)}, \sum_{t \in T} g\left(X_{W^{(k)}}^{t}\right)\right)$, the prosumer temporarily drops out, and is added to the "dropout" set $D$. A high-level description of the iterative process is illustrated in the flowchart of Fig. 2.

Once the iterative procedure terminates, the community manager will allocate power to the prosumers in $D$, in decreasing order of their reached bid based on the resources left. Each prosumer that is accommodated will be transferred to set $P$. The exact auction procedure is described in Algorithm 1 .

Proposition 1. The auction of Algorithm 1, always terminates with a globally feasible allocation.

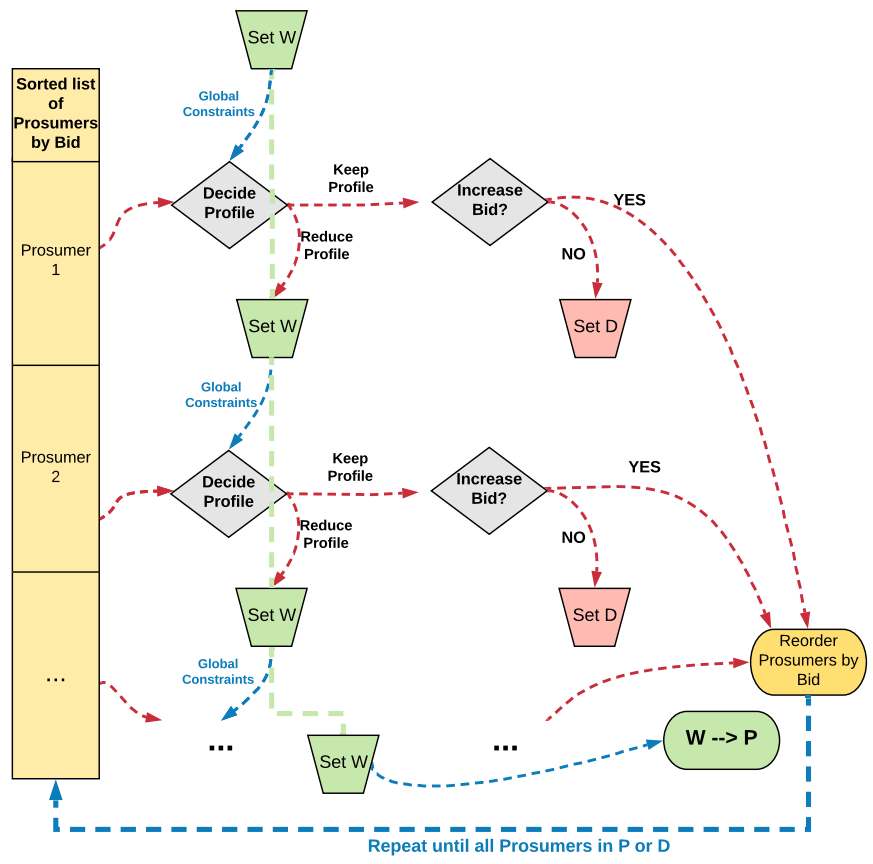

Fig. 2. High-level description of the iterative process of Algorithm 1.

Proof. Notice that, in each iteration of the while loop of Algorithm 1, unless a prosumer enters set $P$, then it either has to increase its bid (i.e., reduce its reward/ increase its bill) or enter set $D$ (lines 17-21). Moreover, by line 18, a prosumer's bid is bounded above. Thus, eventually all prosumers find themselves either in $P$ or $D$, which (by line 4), means that the algorithm always exits the while loop and, consequently, always terminates.

Also, by lines 30-31, after the while loop terminates, a prosumer is added to $P$ if and only if the prosumer is removed from $D$. Thus, when Algorithm 1 terminates, all prosumers are either in set $P$, or have dropped out.

Sets $P$ and $W$ are initialized to the empty set, and a prosumer can only enter set $P$ by first entering set $W$ (lines 16 and 31 of Algorithm 1). However, a prosumer cannot enter set $W$, unless its latest updated vector is consistent with the vectors of prosumers that are already in $W$ (lines 7, 12, 30). Thus, at any point, the profile vectors of prosumers in set $P$ are locally and globally feasible, i.e., $\boldsymbol{X}_{P^{(k)}} \in G, \forall k$.

By combining the three arguments made respectively in the three paragraphs above, it follows that Algorithm 1 always converges and it converges to a globally feasible allocation.

Using this procedure, problem (2) is effectively decomposed into local optimization problems, while global constraints are satisfied by always maintaining a feasible set of profiles.

With respect to the functions $s_{n}(\cdot)$, their choice has been subject to discussion in recent literature on energy communities (e.g. [14], [32], [28]) and relates to how the costs of energy are shared among the community members. Nevertheless, it is easy for the community manager to make sure that the payments received add up to the energy costs, i.e., 


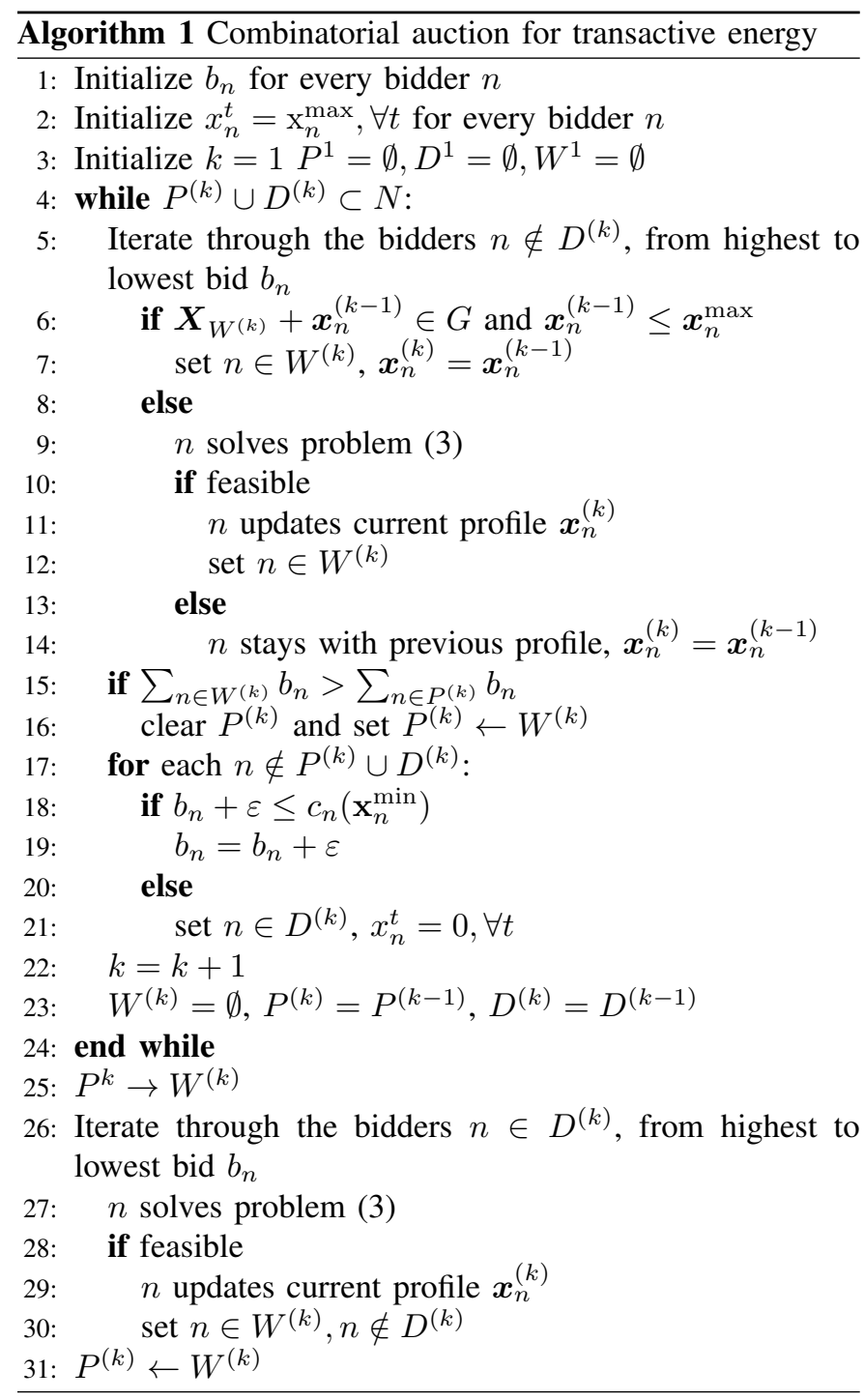

$\sum_{n \in N} s_{n}\left(\boldsymbol{x}_{n}^{(k)}, \sum_{t \in T} g\left(X_{W^{(k)}}^{t}\right)\right)=\sum_{t \in T} g\left(X_{W^{(k)}}^{t}\right)$ as is in fact the case for the schemes proposed in the cited literature. A straightforward choice is to simply pass the wholesale energy price $\lambda^{t}$ of each timeslot, on to the prosumers. In this case $s_{n}(\cdot)$ would be $s_{n}\left(x_{n}^{t}\right)=x_{n}^{t} \lambda^{t}$. Based on this remarks, an important observation is that the proposed scheme is always able to achieve revenue adequacy, i.e., the community manager never runs a budget deficit. This is another advantage over the optimal and incentive-compatible VCG mechanism, since the latter is notorious for suffering from revenue inadequacy issues and is formally stated in the next Proposition.

Proposition 2. By choosing the functions $s_{n}(\cdot)$ such that $\sum_{n \in N} s_{n}\left(\boldsymbol{x}_{n}^{(k)}, \sum_{t \in T} g\left(X_{W^{(k)}}^{t}\right)\right)=\sum_{t \in T} g\left(X_{W^{(k)}}^{t}\right)$, the auction of Algorithm 1, is always revenue adequate for the community manager.

Proof. The total amount of payments received by the community manager is $\sum_{n \in N} b_{n}+$ $\sum_{n \in N} s_{n}\left(\boldsymbol{x}_{n}^{(k)}, \sum_{t \in T} g\left(X_{W^{(k)}}^{t}\right)\right)$ while the total amount of payments made by the community manager for buying the community's energy is $\sum_{t \in T} g\left(X_{W^{(k)}}^{t}\right)$. Therefore, the difference between revenues and costs for the community manager is equal to $\sum_{n \in N} b_{n}$. Observe that the bid of each prosumer is non-decreasing throughout the auction, while the initial bid is non-negative. Thus, the community manager never runs into a budget deficit.

In the proposed combinatorial auction each prosumer $n$ is required to solve problem (3) at each iteration $k$ and respond either with a feasible profile $\boldsymbol{x}_{n}^{(k)}$ or remain with its previous profile $\left(\boldsymbol{x}_{n}^{(k)}=\boldsymbol{x}_{n}^{(k-1)}\right)$ and increase its bid. Let us refer to a "strategy" as the way through which a prosumer decides between these two options throughout the auction's iterations. It is important to note that, at any given iteration, the prosumer cannot know which of the two choices will eventually result in a better outcome for itself since that depends also on the strategies of other prosumers. In technical terms, we say that there is no dominant strategy for a prosumer, i.e., there is not any strategy for prosumer $n$ that achieves a weakly better outcome for $n$ over other strategies. As explained in more detail in [31], because of this property, the concept of dominant-strategy incentive compatibility is no longer relevant, which hinders a game-theoretic analysis of the way that the auction will play out. Thus, the ability to predict the auction's outcome is lost which, in turn, takes away the ability of prosumers to strategize.

Rather, the best that a prosumer can do is choose an (one of many possible) undominated strategy. A prosumer's undominated strategies in our setting are characterized by not opting out unless the prosumer's bid is about to become higher that the prosumer's cost of its minimum possible profile $\mathbf{x}_{n}^{\min }$, i.e., $b_{n}+\varepsilon \geq c_{n}\left(\mathbf{x}_{n}^{\min }\right)$. Based on these properties, we say that the auction is implementable in undominated strategies, i.e., achieves strategy-resistance, which is equivalent to the property of incentive compatibility for such mechanisms [33].

Finally, it is noted that the proposed scheme could in principle be extended in order to cope with use cases where the set of constraints $G$ includes power flow constraints of the physical grid. However, in that case, the constraints $G$ would not depend only on the vector $\boldsymbol{X}_{N}$ of the prosumers' aggregated profiles, but more generally to the particular combination $\left\{\boldsymbol{x}_{n}\right\}_{n \in N}$ of prosumers' profiles. Therefore, solving problem (3) would require gathering all the prosumers' profiles $\boldsymbol{x}_{n}$ as well as the constraints $G$ (including the network topology) to a single point for computations. In practice, this would also require a trusted third party, responsible for gathering the necessary data in a secure way and performing the computations, so as to guarantee data privacy and security. In contrast, when the constraints depend only on the aggregated profile $\boldsymbol{X}_{N}^{t}$ of the community, the assumption of a third party is not necessary.

\section{CAse Study}

In this section we present a case study where we will apply the proposed algorithm and compare it to the optimal benchmark in terms of optimality and scalability. We consider a setting where a community manager is responsible for the dispatch of a set $N$ of prosumers. Each prosumer $n \in N$ features a set $A_{n}$ of flexibility assets, namely an electric 
vehicle (EV), a thermostatically controlled load (TCL) (e.g. air-conditioner), a battery and a small generator. The electricity consumption of asset $m \in A_{n}$, in timeslot $t \in T$ is denoted as $y_{m}^{t}$, while prosumer $n$ 's total consumption in $t$ is $x_{n}^{t}=\sum_{m \in A_{n}} y_{m}^{t}$.

We consider a setting with limited resources. The community is constrained on the amount of total energy consumption by a parameter $\mathrm{R}$, as in

$$
\sum_{n \in N} x_{n}^{t} \leq \mathrm{R}, \forall t \in T
$$

Constraints (4) (one per timeslot), correspond to the globally feasible set $G$, described in Section II.

An asset $m \in A_{n}$ is constrained by an upper and lower power consumption level:

$$
\mathrm{y}_{m}^{\min } \leq y_{m}^{t} \leq \mathrm{y}_{m}^{\max }
$$

and it cannot consume energy before its plug-in time $\mathrm{a}_{m}$ or after its plug-out time $\mathrm{d}_{m}$ :

$$
y_{m}^{t}=0, t<\mathrm{a}_{m}, t>\mathrm{d}_{m}
$$

Also, an EV $i \in A_{n}$ has a certain energy requirement $\mathrm{E}_{i}$ $(\mathrm{kWh})$ that must be fulfilled, while the State-Of-Energy $S O E$ $(\mathrm{kWh})$, of the $\mathrm{EV}$, follows a certain transition function

$$
\begin{gathered}
\mathrm{h}_{i} \sum_{t \in T} y_{i}^{t} \geq \mathrm{E}_{i} \\
S O E_{i}^{t}=S O E_{i}^{t-1}+\mathrm{h}_{i} y_{i}^{t}
\end{gathered}
$$

where parameter $h_{i}$ relates to charging efficiency. When charging at full power capacity $\mathrm{y}_{i}^{\max }$, the EV's energy demand will be fulfilled in a total of $\left\lceil\mathrm{E}_{i} / \mathrm{h}_{i} \mathrm{y}_{i}^{\max }\right\rceil$ timeslots, where $\lceil\cdot\rceil$ denotes rounding to the nearest integer above. When the controlled power is generally lower than $\mathrm{h}_{i} \mathrm{y}_{i}^{\max }$, the EV will suffer an extra waiting time (beyond $\left\lceil\mathrm{E}_{i} / \mathrm{h}_{i} \mathrm{y}_{i}^{\max }\right\rceil$ ). Let the binary variable $u_{i}^{t}$ denote whether in timeslot $t$, EV $i$ still has unsatisfied demand:

$$
u_{i}^{t}= \begin{cases}1, & S O E_{i}^{t}-\mathrm{E}_{i}<0 \\ 0, & S O E_{i}^{t}-\mathrm{E}_{i} \geq 0\end{cases}
$$

Then, the extra waiting time $\theta_{E V}$, (beyond $\left\lceil\mathrm{E}_{i} / \mathrm{h}_{i} \mathrm{y}_{i}^{\max }\right\rceil$ timeslots) that the EV has to wait in order to receive its required energy, is

$$
\theta_{E V}=\sum_{t \in\left[\mathrm{a}_{i},|T|\right]} u_{i}^{t}-\left\lceil\mathrm{E}_{i} / \mathrm{h}_{i} \mathrm{y}_{i}^{\max }\right\rceil-\mathrm{a}_{i}
$$

where in order to define the net waiting time, we subtract the timeslots before the EV's arrival and the timeslots that the EV would wait if it was charging at $\left\lceil\mathrm{E}_{i} / \mathrm{h}_{i} \mathrm{y}_{i}^{\max }\right\rceil$, from the total timeslots that the EV's energy requirement was not satisfied.

For a TCL $j \in A_{n}$ let $F_{j}^{t}$ denote the temperature measured by the TCL's sensor. The transition function of the temperature is defined as:

$$
F_{j}^{t}=F_{j}^{t-1}+\operatorname{ins}_{j}\left(\mathrm{~F}_{e n v}^{t}-F_{j}^{t-1}\right)-\operatorname{con}_{j} y_{j}^{t-1}
$$

where $\mathrm{F}_{e n v}^{t}$ is the environment's temperature, ins $_{j}$ is a parameter related to temperature decay (e.g., insulation) and $\mathrm{con}_{j}$ is a conversion factor (from electrical power to thermal energy). The set points of the TCL controller are denoted as $\mathrm{F}_{j}^{\min }$ for minimum comfortable temperature and $\mathrm{F}_{j}^{\max }$ for maximum comfortable temperature. We assume that when the temperature $F_{j}^{t}$ is within $\left[\mathrm{F}_{j}^{\min }, \mathrm{F}_{j}^{\max }\right]$, the demand is considered satisfied. Let $u_{j}^{t}$ denote whether $F_{j}^{t}$ is beyond the comfort levels in a timeslot that the device is turned on:

$$
u_{j}^{t}= \begin{cases}1, & F_{j}^{t} \notin\left[\mathrm{F}_{j}^{\min }, \mathrm{F}_{j}^{\max }\right] \wedge t \in\left[\mathrm{a}_{j}, \mathrm{~d}_{j}\right] \\ 0, & F_{j}^{t} \in\left[\mathrm{F}_{j}^{\min }, \mathrm{F}_{j}^{\max }\right] \vee t \notin\left[\mathrm{a}_{j}, \mathrm{~d}_{j}\right]\end{cases}
$$

The cost of a TCL, defined as the number of timeslots that the temperature preference is not satisfied, is:

$$
\theta_{T C L}=\sum_{t \in T} u_{j}^{t}
$$

Note that the timeslots in which the TCL is turned off do not count in the device's cost.

A battery $l \in A_{n}$ can charge at timeslot $t$, at a charging rate $y_{l, \mathrm{ch}}^{t}$ or discharge at $y_{l, \mathrm{dis}}^{t}$. The binary variable $u_{l}^{t}$ denotes whether the battery charges $\left(u_{l}^{t}=1\right)$ or discharges at $t$. It is

$$
\begin{aligned}
& 0 \leq y_{l, \mathrm{ch}}^{t} \leq u_{l}^{t} \mathrm{y}_{l}^{\max } \\
& 0 \leq y_{l, \mathrm{dis}}^{t} \leq\left(1-u_{l}^{t}\right) \mathrm{y}_{l}^{\max }
\end{aligned}
$$

The battery's state of charge $S O C_{l}^{t}$ follows the dynamics

$$
S O C_{l}^{t}=S O C_{l}^{t-1}+\mathrm{h}_{l}^{\mathrm{ch}} y_{l, \mathrm{ch}}^{t}-\mathrm{h}_{l}^{\mathrm{dis}} y_{l, \mathrm{dis}}^{t}
$$

where $\mathrm{h}_{l}^{\text {ch }}$ and $\mathrm{h}_{l}^{\text {dis }}$ are parameters for the battery's charging and discharging efficiency. The battery's capacity constraint is modelled as

$$
0 \leq S O C_{l}^{t} \leq 1
$$

The operating cost relates to the battery's degradation, which depends on the amount of charge-discharge cycles, is modelled as

$$
\theta_{\text {Bat }}=\sum_{t \in T} y_{l, \mathrm{dis}^{t}} \operatorname{deg}_{l}
$$

Finally, a microgenerator $g \in A_{n}$ generates $y_{g}^{t}$ in timeslot $t$, and is constrained by (5) and by ramp constraints as

$$
-\operatorname{ramp}_{g} \leq y_{g}^{t}-y_{g}^{t-1} \leq \operatorname{ramp}_{g}, t>1
$$

The generator's cost is approximated by a quadratic function as

$$
\theta_{\text {gen }}=\sum_{t \in T} \mathrm{c}_{g}\left(y_{g}^{t}\right)^{2}
$$

We can then define the cost function of a prosumer $n \in N$, using the above notions for $\theta_{m}$ and a cost parameter $\mathrm{w}_{n}$ :

$$
c_{n}\left(\boldsymbol{x}_{n}\right)=\mathrm{w}_{n} \sum_{m \in A_{n}} \theta_{m}
$$

Finally, we assume that the community buys energy at a pertimeslot price $\lambda^{t}$ and each prosumer $n$ pays proportionally for its hourly consumption, i.e.,

$$
s_{n}\left(x_{n}^{t}\right)=x_{n}^{t} \lambda^{t}
$$

Based on the formulations above, the set of local constraints $L_{n}$ of a prosumer consists of eqs. (5) - (20) together with 
constraints (1). The cost functions are defined by (21) and the global constraints $G$ are defined by (4). Thus, having defined the cost functions and the local and global constraints, the optimal solution of problem (2) can be obtained by having each prosumer communicate its cost functions and local constraints to the community manager. The latter solves an optimization problem (in this case study, a mixed-integer linear program) to determine the profile of every asset of every prosumer. We use this approach as a benchmark to evaluate the proposed method. The simulation setup and results are presented in the next section.

\section{Simulation Setup AND Results}

We simulated the above setting for an horizon of 12 timeslots, and a setup with 15 prosumers, unless stated otherwise. Each prosumer features a TCL, an EV, a battery and a microgenerator i.e., the decision variables for each prosumer are $y_{m}^{t}, x_{n}^{t}, S O E_{i}^{t}, u_{m}^{t}, \theta_{m}, F_{j}^{t}, y_{l, \mathrm{ch}}^{t}, y_{l, \mathrm{dis}}^{t}, S O C_{l}^{t}$. For each test, the results were averaged out over a number of experiments, where in each experiment different parameter values were sampled from random normal distributions. The values or random distributions of setting's parameters are presented in Table II. The outside temperature $\mathrm{F}_{\text {env }}^{t}$ was set between 77 and 110 degrees Fahrenheit (so as to resemble typical summer temperatures). The resource parameters $\mathrm{R}^{t}$ were sampled from a normal distribution with standard deviation of 1 , and an average value of $\mathrm{r}|N| \max \left(\mathrm{y}_{m}^{\max }\right)$, where $\mathrm{r}$ is a percentage value. The lower the $r$, the less the available resources. Unless stated otherwise, parameter $r$ was set to the minimum value for which problem (2) remains feasible.

TABLE II

VALUES/DISTRIBUTIONS OF SETTING'S PARAMETERS

\begin{tabular}{|c|c|c|c|c|}
\hline Parameter & Comments & Value & Average Value & Standard deviation \\
\hline $\mathrm{y}_{m}^{\min }$ & $\forall m$ & 0 & - & - \\
\hline $\mathrm{y}_{m}^{\max }$ & for EVs & - & 3 & 0.1 \\
\hline $\mathrm{y}_{m}^{\max }$ & for TCLs & - & 4 & 0.1 \\
\hline $\mathrm{y}_{m}^{\max }$ & for batteries & - & 2 & 0.1 \\
\hline $\mathrm{y}_{m}^{\max }$ & for generators & - & 1.5 & 0.2 \\
\hline $\mathrm{a}_{m}$ & for EVs & - & 4 & 2 \\
\hline $\mathrm{a}_{m}$ & for TCLs & 0 & - & - \\
\hline $\mathrm{d}_{m}$ & $\forall m$ & $\bar{T} \mid$ & - & - \\
\hline $\mathrm{h}_{i}$ & - & - & 1 & 0.1 \\
\hline $\mathrm{h}_{l}^{\mathrm{ch}}$ & - & $1 / 3 y_{m}^{\max }$ & - & - \\
\hline $\mathrm{h}_{l}^{\text {dis }}$ & - & $1 / 2 y_{m}^{\max }$ & - & - \\
\hline $\mathrm{E}_{i}$ & - & - & 8 & 1 \\
\hline ins $_{j}$ & - & 0.05 & - & - \\
\hline $\operatorname{con}_{j}$ & - & 3.5 & - & - \\
\hline $\operatorname{deg}_{l}$ & - & - & 0.01 & 0.003 \\
\hline $\mathrm{c}_{g}$ & - & - & 0.1 & 0.02 \\
\hline $\operatorname{ramp}_{g}$ & - & - & 0.5 & 0.2 \\
\hline $\mathrm{F}_{j}^{\min }$ & - & 73 & - & - \\
\hline $\mathrm{F}_{j}^{\max }$ & - & 80 & - & - \\
\hline $\mathrm{w}_{n}$ & $\forall n$ & - & 1 & 0.2 \\
\hline$\lambda^{t}$ & $\forall t$ & - & 0.09 & 0.02 \\
\hline
\end{tabular}

\section{A. Scalability}

Compared to the optimal and incentive compatible VCG mechanism, the proposed approach sacrifices optimality guarantees, in order to achieve faster computational time and scalability. The computational time of VCG, rises quickly with the

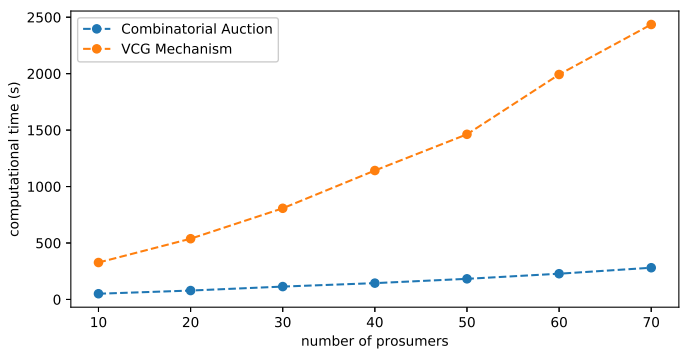

Fig. 3. Scalability of the optimal VCG approach compared to the proposed scheme

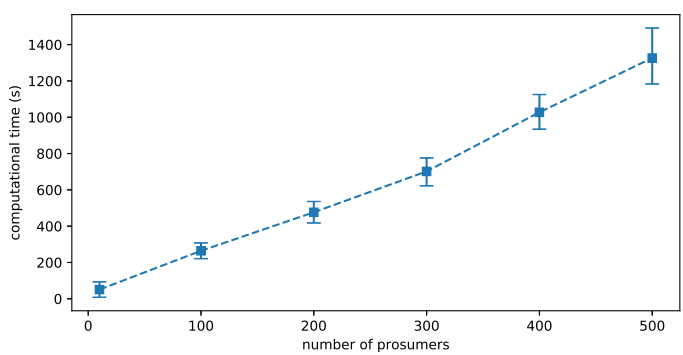

Fig. 4. Scalability of the proposed scheme to large numbers of prosumers

number of prosumers as can be observed in Fig. 3, in contrast to the proposed approach. Moreover, the proposed approach scales remarkably well to large numbers of prosumers as can be seen in Fig. 4. The experiments were run in Python 3 environment, using CPLEX. The hardware was an i5, $2.7 \mathrm{GHz}$ computer with 8GB RAM and two cores.

\section{B. Efficiency vs Computational time trade-off}

A design choice of the auction is the step $\varepsilon$ with which the prosumers' bids are increased. We simulated the setting using different values for $\varepsilon$. For each value of $\varepsilon$, a number of 30 experiments were run, where in each experiment a different combination of system parameters was used, by sampling from the parameter distributions of Table II. For each experiment the resulted computational time and system cost were scattered on a $2 \mathrm{D}$ plane, using a different color depending on $\varepsilon$. The result is depicted in Fig. 5. A general trend is observed, that lower choices of step $\varepsilon$ result in higher computational times.

Interestingly, the auction's efficiency does not seem to be improved by further reducing the step $\varepsilon$, after a certain point, and it even deteriorates. To demonstrate this on the figure, a second degree polynomial was fitted to the resulting points and is shown on the same graph with a red line. The best choice in terms of efficiency is a step around 0.1. Another observation is that by using a higher step (e.g. $\varepsilon=0.4$ ), the computational time can be shortened, usually without significant loss of efficiency on average. However, a choice of such a large step, comes with a risk of severe efficiency loss, as can be seen by the purple outliers on the upper left part of the figure. 


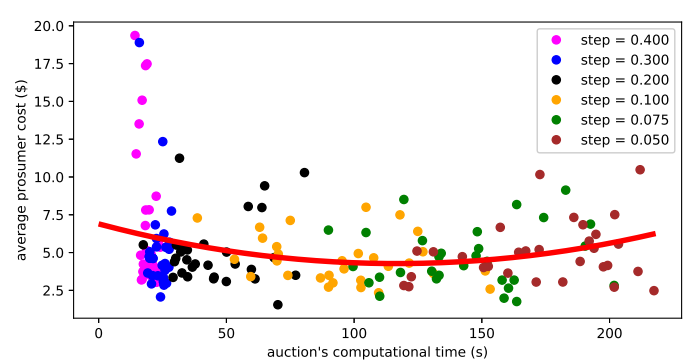

Fig. 5. Computational time and efficiency of the proposed scheme for different values of the bid-increasing step $\varepsilon$

\section{Game-theoretic aspects}

In the proposed scheme each prosumer $n$ is required to solve problem (3) at each iteration $k$ and respond either with a feasible profile $\boldsymbol{x}_{n}^{(k)}$ or remain with its previous profile $\left(\boldsymbol{x}_{n}^{(k)}=\boldsymbol{x}_{n}^{(k-1)}\right)$ and increase its bid. In this subsection, we model the prosumer's choice by implementing a threshold $\overline{\theta_{n}}$, where if $\sum_{m \in A_{n}} \theta_{m}^{(k)} \leq \overline{\theta_{n}}$, the prosumer accepts the solution. If $\sum_{m \in A_{n}} \theta_{m}>\overline{\theta_{n}}$, then the prosumer opts for passing, and prefers to increase its bid rather than accept this solution. Intuitively, if $\overline{\theta_{n}}$ is very high, the prosumer might accept a very unfavorable profile at some iteration and it will not be able to escape from it in later iterations due to the last constraint of problem (3). Formally, the aforementioned threshold is implemented by adding constraint

$$
\sum_{m \in A_{n}} \theta_{m} \leq \overline{\theta_{n}}
$$

to each prosumer's local set of constraints $L_{n}$.

From the prosumer's perspective, its payoff depends on its own selection $\overline{\theta_{n}}$ as well as on the thresholds of the other prosumers. Thus, a game is formed, where the players are the prosumers, the set of strategies available to each player is the choice of $\overline{\theta_{n}}$, the set of payoffs are the local costs $c_{n}\left(\boldsymbol{x}_{n}^{*}\right)-b_{n}^{*}$, where $\boldsymbol{x}_{n}^{*}$ is the allocation resulted from the auction and $b_{n}^{*}$ is the final bid of prosumer $n$ (i.e., the amount that $n$ is required to pay for its allocation).

We model a case where each prosumer can choose among 6 available choices for $\overline{\theta_{n}}$, that is the set of strategies is $S=\overline{\theta_{n}}=\{2,4,6,8,10,12\}$. In order to calculate an equilibrium point, we simulated a best-response procedure, described in Algorithm 2. For the experiments tested, Algorithm 2 converges to an equilibrium point only after a few iterations. An indicative case is are shown in Fig. 6.

\section{Efficiency of equilibria}

In this test, we evaluate the efficiency of the proposed auction, compared to the optimal solution, which was calculated by centrally solving problem (2), assuming that all local prosumer parameters are known. The solution was obtained using the CPLEX solver, where constraints (9) and (12) were relaxed using the big-M method as in [34].

We assessed the efficiency of the proposed combinatorial auction, in terms of the system cost $\sum_{n \in N} c_{n}\left(\boldsymbol{x}_{n}\right)+$
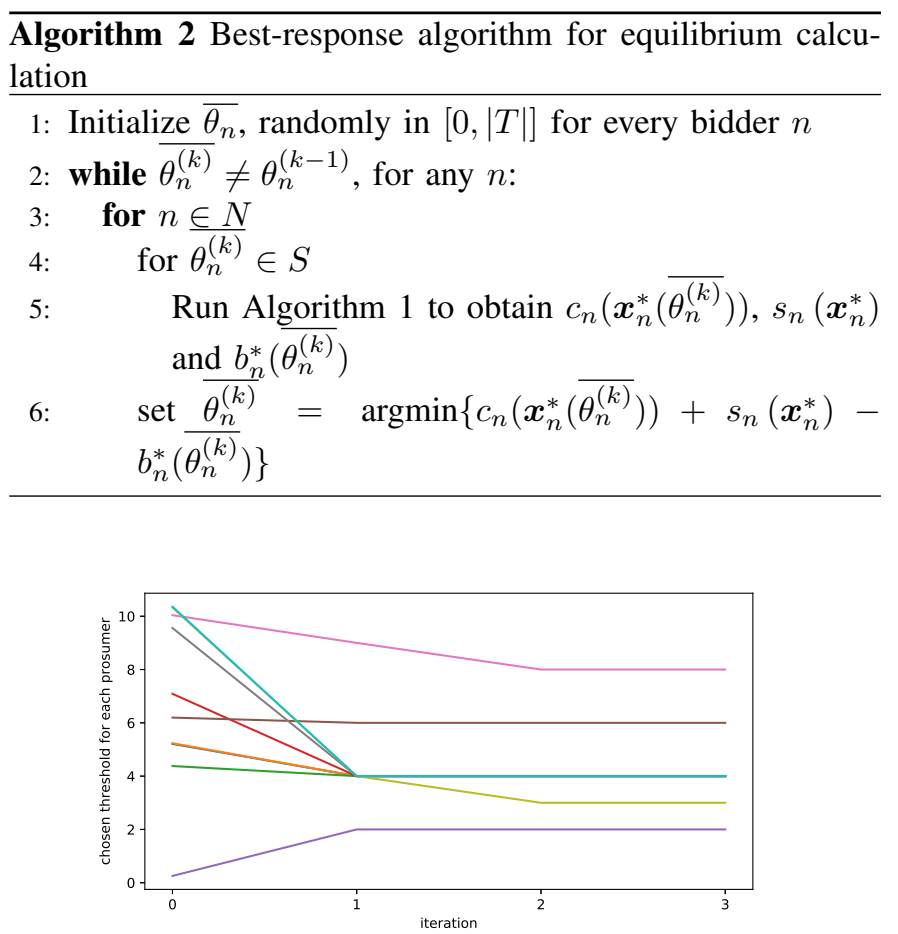

Fig. 6. prosumers' strategies at each iteration of the auction (one line per prosumer)

$\sum_{t \in T} g\left(X_{W}^{t}\right)$, in two cases. In the first case an arbitrary value for $\theta_{n}$ (within $[0,14]$ ) was chosen randomly for each prosumer. In the second case, the equilibrium values $\overline{\theta_{n}^{\mathrm{eq}}}$, as calculated by Algorithm 2, were used. The efficiency was tested for various values of parameter $r$ (i.e. the parameter that defines the level of available resources $R$ as explained at the beginning of this section). The results were plotted against the optimal benchmark. Fig. 7 depicts the results, where for each value of $r$, the results were averaged out over a number of experiments.

Two important observations can be made by Fig. 7. On the positive side, when equilibrium values $\overline{\theta_{n}^{\mathrm{eq}}}$ were used for the thresholds, the efficiency was better than in the case of random threshold values. This means that, when prosumers try to improve their own benefit by learning a good $\overline{\theta_{n}}$, they simultaneously improve the auction's efficiency, i.e., the individual incentive and the social objective are aligned. On the negative side, in settings with very scarce resources (lower $r$ ) the auction suffers a non-negligible efficiency loss. Nevertheless, the only way to achieve the optimal value in a setting with strategic users, is to employ the VCG mechanism, which suffers from revenue adequacy issues and, as shown in Fig.3, also from limited scalability.

\section{CONClusions And Future Work}

In this paper we presented a combinatorial auction through which a community manager can make energy allocation decisions for a community of prosumers in a setting with resource constraints and strategic prosumer agents. The scheme scales very well to large numbers of prosumers, in contrast to the optimal Vickrey-Clarke-Groves mechanism. We evaluated the scheme using a case study, also considering game-theoretic 


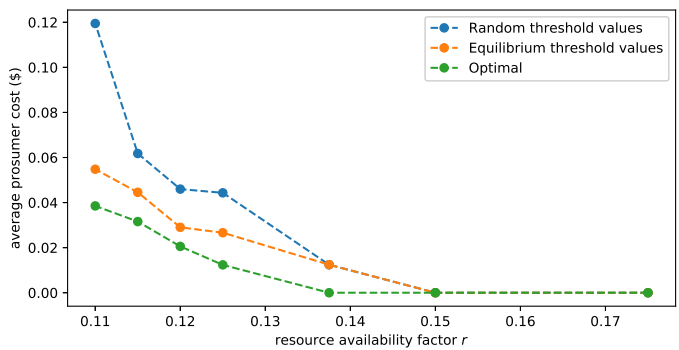

Fig. 7. Efficiency of the proposed scheme for two cases of threshold selection and for various cases of resource availability, compared to the optimal solution

aspects of prosumer participation. An equilibrium analysis was performed and the efficiency of equilibria was evaluated. The design of the auction does not leave much room for strategizing, while simulations show that the prosumers, by trying to strategize, only improve the auction's efficiency, which implies an alignment of prosumer incentives with the social objective.

While the results for this case study are positive, further research needs to be done on whether an incentive alignment can be achieved in different use cases. Most importantly, in this paper we have considered deterministic prosumer models and future work should also consider the issue of uncertainty and conduct simulations in settings with learning algorithms employed in each prosumer's side.

\section{REFERENCES}

[1] J. E. Contreras-Ocaña, Y. Chen, U. Siddiqi, and B. Zhang, "Nonwire alternatives: An additional value stream for distributed energy resources," IEEE Transactions on Sustainable Energy, vol. 11, no. 3, pp. 1287-1299, 2020.

[2] M. Caramanis, E. Ntakou, W. W. Hogan, A. Chakrabortty, and J. Schoene, "Co-optimization of power and reserves in dynamic t d power markets with nondispatchable renewable generation and distributed energy resources," Proceedings of the IEEE, vol. 104, no. 4, pp. 807-836, 2016.

[3] A. Papavasiliou, "Analysis of distribution locational marginal prices," IEEE Transactions on Smart Grid, vol. 9, no. 5, pp. 4872-4882, 2018.

[4] R. A. Verzijlbergh, L. J. De Vries, and Z. Lukszo, "Renewable energy sources and responsive demand. do we need congestion management in the distribution grid?" IEEE Transactions on Power Systems, vol. 29, no. 5, pp. 2119-2128, 2014.

[5] I. Dukovska, N. G. Paterakis, and H. J. G. Slootweg, "Coordination for prosumers' electricity trading agents via distributed optimization," in 2019 International Conference on Smart Energy Systems and Technologies (SEST), 2019, pp. 1-6.

[6] J. Hu, G. Yang, H. W. Bindner, and Y. Xue, "Application of networkconstrained transactive control to electric vehicle charging for secure grid operation," IEEE Transactions on Sustainable Energy, vol. 8, no. 2, pp. 505-515, 2017

[7] A. Falsone, K. Margellos, and M. Prandini, "A decentralized approach to multi-agent milps: Finite-time feasibility and performance guarantees," Automatica, vol. 103, pp. 141 - 150, 2019.

[8] J. R. Vázquez-Canteli and Z. Nagy, "Reinforcement learning for demand response: A review of algorithms and modeling techniques," Applied energy, vol. 235, pp. 1072-1089, 2019.

[9] F. Ruelens, B. J. Claessens, S. Vandael, B. De Schutter, R. Babuška, and R. Belmans, "Residential demand response of thermostatically controlled loads using batch reinforcement learning," IEEE Transactions on Smart Grid, vol. 8, no. 5, pp. 2149-2159, 2017.

[10] A. Cully, J. Clune, D. Tarapore, and J.-B. Mouret, "Robots that can adapt like animals," Nature, vol. 521, no. 7553, pp. 503-507, 2015.
[11] B. Baker, I. Kanitscheider, T. Markov, Y. Wu, G. Powell, B. McGrew, and I. Mordatch, "Emergent tool use from multi-agent autocurricula," arXiv preprint arXiv:1909.07528, 2019.

[12] Y. Wang, S. Wang, and L. Wu, "Distributed optimization approaches for emerging power systems operation: A review," Electric Power Systems Research, vol. 144, pp. 127 - 135, 2017.

[13] J. Guerrero, A. C. Chapman, and G. Verbič, "Decentralized p2p energy trading under network constraints in a low-voltage network," IEEE Transactions on Smart Grid, vol. 10, no. 5, pp. 5163-5173, 2019.

[14] F. Moret and P. Pinson, "Energy collectives: A community and fairness based approach to future electricity markets," IEEE Transactions on Power Systems, vol. 34, no. 5, pp. 3994-4004, 2019.

[15] C. Orozco, S. Lilla, A. Borghetti, F. Napolitano, and F. Tossani, "An admm approach for day-ahead scheduling of a local energy community," in 2019 IEEE Milan PowerTech, 2019, pp. 1-6.

[16] P. Samadi, A. Mohsenian-Rad, R. Schober, V. W. S. Wong, and J. Jatskevich, "Optimal real-time pricing algorithm based on utility maximization for smart grid," in 2010 First IEEE International Conference on Smart Grid Communications, 2010, pp. 415-420.

[17] S. Bahrami, M. H. Amini, M. Shafie-khah, and J. P. S. Catalão, "A decentralized electricity market scheme enabling demand response deployment," IEEE Transactions on Power Systems, vol. 33, no. 4, pp. 4218-4227, 2018.

[18] G. Tsaousoglou, K. Steriotis, and E. Varvarigos, "A stochastic approximation method for price-based assignment of electric vehicles to charging stations," in 2019 International Conference on Smart Energy Systems and Technologies (SEST), 2019, pp. 1-6.

[19] H.-M. Chung, S. Maharjan, Y. Zhang, and F. Eliassen, "Distributed deep reinforcement learning for intelligent load scheduling in residential smart grids," 2020.

[20] Z. Pan, T. Yu, J. Li, K. Qu, L. Chen, B. Yang, and W. Guo, "Stochastic transactive control for electric vehicle aggregators coordination: A decentralized approximate dynamic programming approach," IEEE Transactions on Smart Grid, vol. 11, no. 5, pp. 4261-4277, 2020.

[21] Y. Ye, D. Qiu, J. Li, and G. Strbac, "Multi-period and multi-spatial equilibrium analysis in imperfect electricity markets: A novel multiagent deep reinforcement learning approach," IEEE Access, vol. 7, pp. 130 515-130529, 2019.

[22] K. Steriotis, K. Smpoukis, N. Efthymiopoulos, G. Tsaousoglou, P. Makris, and E. Varvarigos, "Strategic and network-aware bidding policy for electric utilities through the optimal orchestration of a virtual and heterogeneous flexibility assets' portfolio," Electric Power Systems Research, vol. 184, 2020.

[23] C. P. Mediwaththe, M. Shaw, S. Halgamuge, D. B. Smith, and P. Scott, "An incentive-compatible energy trading framework for neighborhood area networks with shared energy storage," IEEE Transactions on Sustainable Energy, vol. 11, no. 1, pp. 467-476, 2020.

[24] P. Samadi, H. Mohsenian-Rad, R. Schober, and V. W. S. Wong, "Advanced demand side management for the future smart grid using mechanism design," IEEE Transactions on Smart Grid, vol. 3, no. 3, pp. 1170-1180, 2012.

[25] K. Seklos, G. Tsaousoglou, K. Steriotis, N. Efthymiopoulos, P. Makris, and E. Varvarigos, "Designing a distribution level flexibility market using mechanism design and optimal power flow," in 2020 International Conference on Smart Energy Systems and Technologies (SEST), 2020, pp. 1-6.

[26] G. Tsaousoglou, K. Steriotis, N. Efthymiopoulos, P. Makris, and E. Varvarigos, "Truthful, practical and privacy-aware demand response in the smart grid via a distributed and optimal mechanism," IEEE Transactions on Smart Grid, pp. 1-1, 2020.

[27] R. Deng, Z. Yang, J. Chen, N. R. Asr, and M. Chow, "Residential energy consumption scheduling: A coupled-constraint game approach," IEEE Transactions on Smart Grid, vol. 5, no. 3, pp. 1340-1350, 2014.

[28] G. Tsaousoglou, K. Steriotis, N. Efthymiopoulos, K. Smpoukis, and E. Varvarigos, "Near-optimal demand side management for retail electricity markets with strategic users and coupling constraints," Sustainable Energy, Grids and Networks, vol. 19, 2019.

[29] G. Tsaousoglou, J. S. Giraldo, P. Pinson, and N. G. Paterakis, "Mechanism design for fair and efficient dso flexibility markets," IEEE Transactions on Smart Grid, pp. 1-1, 2020.

[30] B. Biegel, P. Andersen, J. Stoustrup, and J. Bendtsen, "Congestion management in a smart grid via shadow prices," IFAC Proceedings Volumes, vol. 45, no. 21, 2012.

[31] M. Babaioff, R. Lavi, and E. Pavlov, "Single-value combinatorial auctions and algorithmic implementation in undominated strategies," $J$. $A C M$, vol. 56, no. 1, 2009. 
[32] G. Tsaousoglou, N. Efthymiopoulos, P. Makris, and E. Varvarigos, "Personalized real time pricing for efficient and fair demand response in energy cooperatives and highly competitive flexibility markets," Jour. of Modern Power Syst. and Clean Energy, vol. 7, pp. 151 - 162, 2019.

[33] M. O. Jackson, "Implementation in Undominated Strategies: A Look at Bounded Mechanisms," The Review of Economic Studies, vol. 59, no. 4, pp. 757-775, 091992.

[34] G. Tsaousoglou, P. Pinson, and N. G. Paterakis, "Max-min fairness for demand side management under high res penetration: Dealing with undefined consumer valuation functions," in 2020 International Conference on Smart Energy Systems and Technologies (SEST), 2020, pp. 1-6. 